\section{Scania beschichtet Lkw-Fahrerhäuser in Schweden mit Pulverfüller}

D ürr baut für den zum VW-Konzern gehörenden Lkw-Hersteller Scania Lackierlinien für Fahrerhäuser in Oskarshamn (Schweden) und Sao Paulo (Brasilien). Bei der Anlage in Schweden kommt in der Füllerlinie Pulverlack zum Einsatz. Die Pulverlackieranlage ist mit einer Pulverrückgewinnung ausgestattet, in der nicht auf dem Fahrerhaus haftendes Pulver gesammelt, aufbereitet und zur Weiterverwendung bereitgestellt wird. Der Auftrag des Pulverlacks erfolgt mit 14 Dürr-Lackierrobotern der Typen EcoRP L033, ECoRP L133 und EcoRP E033. Bei der Auftragsvergabe im zweiten Quartal 2012 hat Dürr von seiner Erfahrung bei der
Applikation von Pulverlack profitiert. Auch die Tatsache, dass bereits Fahrerhäuser unter anderem für Scania, Freightliner und Volvo in Lackierlinien von Dürr beschichtet werden, schlägt hier positiv zu Buche. In Brasilien, wo Dürr bereits 1964 seine erste Auslandsgesellschaft gegründet hat, installiert Dürr eine konventionelle Decklacklinie für Nasslack mit zwei Lackierrobotern des Typs EcoRP E133. Diese ist ausgelegt für eine Kapazität von sechs Fahrerhäusern pro Stunde, während die Linie in Schweden 20 Einheiten pro Stunde lackiert. Bei beiden Anlagen gehört die Pulver- beziehungsweise Lackversorgung zum Auftragsumfang.

\title{
Yaskawa eröffnet neue Europa-Firmenzentrale
}

$\mathrm{D}$ er Industrieroboterhersteller Yaskawa hat am 23. Oktober 2012 seine neue Firmenzentrale in Allershausen bei München eröffnet. Rund 12,5 Mio. Euro investierte der Konzern in den Neubau. Die neue Firmenzentrale erstreckt sich auf circa 14000 Quadratmetern Geländefläche. Rund 50 neue Mitarbeiter wurden von Yaskawa zusätzlich eingestellt. "Allers- hausen ist unser Kompetenz-Zentrum für die Emea-Länder", charakterisierte Manfred Stern, Geschäftsführer von Yaskawa Europe (President \& COO), die Rolle des Standortes anlässlich der Eröffnung. Von Allershausen aus betreut die Robotics Division von Yaskawa Europe die Märkte Europa, Afrika, Mittlerer Osten sowie den Bereich der früheren Sowjetunion.

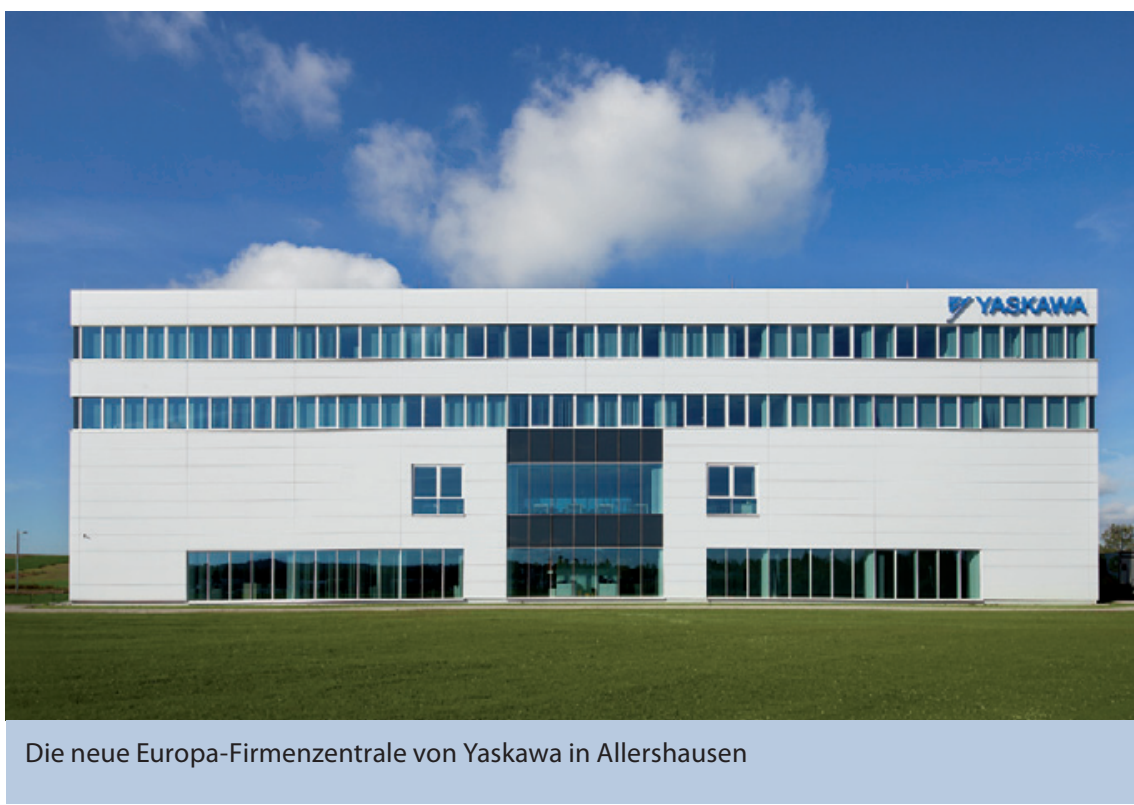

\section{Jahre Steinle-Pumpen}

$D^{\prime}$ Steinle Industriepumpen $\mathrm{GmbH}$, Düsseldorf, feiert im Dezember 2012 das 20-jährige Firmenjubiläum. Am 1. Dezember 1992 gründete Michael Steinle die Michael Steinle, Apparatebau und Verfahrenstechnik. Das Unternehmen startete mit der Entwicklung und dem Vertrieb einer speziellen, druckluftbetriebenen Kolbenmembranpumpe für die Filterpressenbeschickung. 1995 hat Steinle die Vertretung für Tapflo AB aus Schweden für Deutschland übernommen. Tapflo fertigt Druckluftmembranpumpen, die das Produktprogramm in idealer Weise ergänzte. Auf der Basis dieser Pumpe entwickelte Steinle eine kompakte, druckübersetzte Pumpe für die Beschickung von Filterpressen. Heute macht das Geschäft mit Druckluftmembranpumpen 80 Prozent des Unternehmensumsatzes aus. Seit 2001 firmiert das Unternehmen unter Steinle Industriepumpen $\mathrm{GmbH}$. Steinle vertritt mittlerweile den französischen Schlauchpumpenhersteller Albin. In beiderseitiger Kooperation hat die Tapflo-Gruppe die Vertretung für Steinle-Pumpen in Europa übernommen. Mit zehn Mitarbeitern erwirtschaftet Steinle 2012 3,7 Mio. Euro Umsatz.

\section{Termine}

Solingen

17.-18.12.2012

Grundlagen der Korrosion und Korrosionsprüftechnik

Veranstalter: Institut für Galvanound Oberflächentechnik Solingen $\mathrm{GmbH} \&$ Co.KG www.igos.de Tel. 0212 2494-700

München

14.-15.3.2013

EPS - 32. Fachtagung Elektrostatisches Pulverbeschichten Veranstalter: JOTLive www.JOTlive.de Tel. 0611 7878-675 